\title{
A Research Survey of Behavioral Biases of Investment Finance
}

\author{
Xiaofeng $\mathrm{Wu}$ \\ College of Economics and Management, Huaiyin Normal University \\ Huaian 223300, China \\ E-mail: xiashiliang2006@126.com
}

\begin{abstract}
Because psychology systematically explores human judgment, behavior, and well-being, it can teach us important facts about how humans differ from traditional economic assumptions. In this essay I discuss a selection of psychological findings relevant to economics. Standard economics assumes that each person has stable, well-defined preferences, and that she rationally maximizes those preferences. Even if we are willing to modify our familiar assumptions about preferences, or allow that people make systematic errors in their attempts to maximize those preferences, it is sometimes misleading to conceptualize people as attempting to maximize well-defined, coherent, or stable preferences.
\end{abstract}

Keywords: Traditional economics, Systematic errors, Standard economics, Behavioral biases

\section{Introduction}

Because psychology systematically explores human judgment, behavior, and well-being, it can teach us important facts about how humans differ from traditional economic assumptions. In this essay I discuss a selection of psychological findings relevant to economics. There are two basic components of the standard economics model of the individual: That she has stable, well-defined preferences, and that she rationally maximizes those preferences. Given some choice set $\mathrm{X}$, a person is assumed to "Max $\mathrm{x} \in \mathrm{X} \mathrm{U}(\mathrm{x})$." Psychological research can be roughly categorized by how radically it challenges this model, and by the nature of the modifications implied.

\section{Biases in judgment}

Economists have traditionally assumed that, when faced with uncertainty, people correctly form their subjective probabilistic assessments according to the laws of probability. But researchers have documented many systematic departures from rationality in judgment under uncertainty. A feel for this research is well expressed in Tversky and Kahneman (1974), which serves as a de facto introduction to Kahneman, Slovic and Tversky (1982), an influential volume containing many of the major contributions to behavioral research on heuristics and biases: How do people assess the probability of an uncertain event or the value of an uncertain quantity? This article shows that people rely on a limited number of heuristic principles which reduce the complex tasks of assessing probabilities and predicting values to simpler judgmental operations. In general, these heuristics are quite useful, but sometimes they lead to severe and systematic errors. The subjective assessment of probability resembles the subjective assessment of physical quantities such as distance or size. These judgments are all based on data of limited validity, which are processed according to heuristic rules. For example, the apparent distance of an objects determined in part by its clarity. The more sharply the object is seen, the closer it appears to be. This rule has some validity, because in any given scene the more distant objects are seen less sharply than nearer objects. However, the reliance on this rule leads to systematic errors in the estimation of distance. Specifically, distances are often overestimated when visibility is poor, because the contours of objects are blurred. On the other hand, distances are often underestimated when visibility is good because the objects are seen sharply. Thus, the reliance on clarity as an indication of distance leads to common biases. Such biases are also found in the intuitive judgment of probability. Behavioral research on judgment under uncertainty teaches us that people are not nearly as rational as economists assume. But it teaches us much more. In this passage, the word "error" does not appear without the word "systematic". This literature does not merely teach us that people aren't perfectly rational; it proposes specific ways that judgment departs from the rational model. While research on heuristics and biases is not as amenable to incorporation into mainstream formal economics as the material presented in the previous section, this literature already contains some clear and general insights. For the remainder of this section, I describe some of this research. I concentrate on just a few particular heuristics and biases, and then more quickly outline some others. I conclude by discussing briefly some of the evidence for how people do and don't learn to correct biases.

\section{The Representativeness Heuristic}

We think a person is more likely to be a member of some group if that person is similar to the typical member of that 
group. If a man behaves more like a criminal (shifty eyes, etc.), then we think it is more likely he is a criminal. This simple intuition is, of course, captured by Bayes Law.

Research on the representativeness heuristic, however, demonstrates that people tend to over-use "representativeness" in assessing probabilities. Bayes' Law tells us that our assessment of likelihoods should combine representativeness with base rates (the percentage of the population falling into various groups). Yet people under-use base-rate information in forming their judgments. If we see somebody who looks like a criminal, our assessment of the probability that he is a criminal tends to under-use knowledge about the percentage of people who are criminals. Similarly, if a certain medical test always comes out positive among people with a rare disease, and only occasionally among people without the disease, people will tend to exaggerate the likelihood of having the disease given a positive result. Given the rarity of the disease, the total number of false positives may be far greater than the number of true positives.

\section{The Law of Small Numbers}

A phenomenon related to the under-use of base rates is "the law of small numbers": People exaggerate how often to which a small group will closely resemble the parent population or underlying probability distribution that generates the group. We expect even small classes of students to contain very close to the typical distribution of smart ones and personable ones. Likewise, we underestimate how often a good financial analyst will be wrong a few times in a row, and how often a clueless analyst will be right a few times in a row. By the law of small numbers, people expect close to the same probability distribution of types in small groups as they do in large groups. For example, it is much less likely that at least $80 \%$ of 20 coin flips will come up heads than that at least $80 \%$ of 5 coin flips will come up heads (about $1 \%$ and 19\%, respectively); however, people tend to view these as comparably likely. For example, Kahneman and Tversky asked undergraduates the following question: A certain town is served by two hospitals. In the larger hospital about 45 babies are born each day, and in the smaller hospital about 15 babies are born each day. As you know, about 50 percent of all babies are boys. However, the exact percentage varies from day to day. Sometimes it may be higher than 50 percent, sometimes lower. For a period of 1 year, each hospital recorded the days on which more than 60 percent of the babies born were boys. Which hospital do you think recorded more such days? Twenty-two percent of the subjects said that they thought that it was more likely that the larger hospital recorded more such days, and $56 \%$ said that they thought the number of days would be about the same. Only $22 \%$ of subjects answered correctly that the smaller hospital would report more such days. This is the same fraction as guessed exactly wrong. Apparently, the subjects simply did not see the relevance of the number of child births per day. While people believe in the law of small numbers, they apparently don't believe in the law of large numbers: While we exaggerate the resemblance of small samples to the overall population, we underestimate the resemblance that large samples will have to the overall population.

The law of small numbers implies that people exaggerate the likelihood that a short sequence of flips of a fair coin will yield roughly the same number of heads as tails. What is commonly known as "the gambler's fallacy" is a manifestation of this bias: If a fair coin has not come up tails for a while, then on the next flip it is "due" for a tails, because a sequence of flips of a fair coin ought to include about as many tails as heads. When the underlying probability distribution generating observed sequences is uncertain, the fallacy leads people to over-infer the probability distribution from short sequences. Because we exaggerate how likely it is that a bad financial analyst making three predictions will be wrong at least once, we exaggerate the likelihood that an analyst is good if she is right three times in a row.

\section{Belief Perseverance and Confirmatory Bias}

Once forming strong hypotheses, people often are less attentive to relevant new information supporting or contradicting their hypotheses. For instance, once you become convinced that one investment strategy is more lucrative than another, you may simply stop paying attention to the feedback you get. This can often lead people to maintain a hypothesis formed on weak evidence, even if later evidence should lead them to reject earlier beliefs. A particularly elegant demonstration of "anchoring" is found in Bruner and Potter. About 90 subjects were shown blurred pictures that were gradually brought into sharper focus. Different subjects began viewing the pictures at different points in the focusing process, but the pace of the focusing process and final degree of focus were identical for all subjects. Strikingly, of those subjects who began their viewing at a severe-blur stage, less than a quarter eventually identified the pictures correctly, whereas over half of those who began viewing at a light-blur stage were able to correctly identify the pictures. Bruner and Potter (1964, p. 424) conclude that "Interference may be accounted for partly by the difficulty of rejecting incorrect hypotheses based on substandard cues." That is, people who use weak evidence to form initial hypotheses have difficulty correctly interpreting subsequent, better information that contradicts those initial hypotheses. This form of anchoring does not necessarily imply that people misinterpret additional evidence, only that they ignore additional evidence. While such anchoring is potentially quite important, psychological evidence reveals a stronger and more provocative phenomenon: People tend to misread evidence as additional support for initial hypotheses. 


\section{Conclusions}

Mainstream economics employs a powerful combination of methods: methodological individualism, mathematical formalization of assumptions, logical analysis of what conclusions follow from those assumptions, and sophisticated empirical field testing. I believe these methods are tremendously useful, and should not be abandoned. But these methods often raise a barrier to incorporating psychological insights into economics: While psychology investigates humans in all their richness, economics requires models that are not so rich as to retard the process of drawing out their economic implications. For a discipline such as economics that places a high premium on the logic and precision of arguments and the quantification of evidence, incorporating all facets of human nature is neither attainable nor desirable.

As messy as complicating our familiar model of humans will be, however, it is not legitimate for economics to continue to ignore psychological research. Many psychological findings are robust enough, tractable enough, and of enough potential economic importance that we ought begin to integrate them into economics. Other findings raise more fundamental challenges, and incorporating them into economic research will take longer. But even in these cases, economists ought to become aware of the shortcomings of our models, regret these shortcomings, and keep our eyes open for ways to remedy them. Most importantly, we must abandon meta-arguments about whether it is "possible" that psychologists have identified economically relevant departures from rationality, self interest, and other familiar assumptions. Of course it is possible, and in fact it is true. In this essay, I review what research shows some of those departures to be.

\section{References}

Adams, J. S. (1963). Wage Inequities, Productivity and Work Quality. Industrial Relations, 3: 9-16.

Ainslie, G.. (2005). Specious reward: A behavioral theory of impulsiveness and impulse control. Psychological Bulletin, 82(4): 463-496.

Battalio, R. C., J. H. Kagel and K. Jiranyakul. (1990). Testing between Alternative Models of Choice under Uncertainty: Some Initial Results. Journal of Risk and Uncertainty, 3(1): 25-50.

Baumann, A. O., R. B. Deber and G. G. Thompson. (2007). Overconfidence among physicians and nurses: The "micro-certainty, macro-uncertainty" phenomenon. Social Science \& Medicine, 32(2): 167-174.

Fehr, E. and P. Zych. (2004). The Power of Temptation: Irrationally Myopic Excess Consumption in an Addiction Experiment. Mimeo (September).

Fellner, W. (1961). Distortion of subjective probabilities as a reaction to uncertainty. Quarterly Journal of Economics, 75: 670-694.

Kagel, J. and A. Roth, Eds. (2006). Handbook of Experimental Economics. Princeton, Princeton University Press.

Kahn, A. and T. Tice (2003). Returning a Favor and Retaliating Harm: The Effects of Stated Intentions and Actual Behavior. Journal of Experimental Social Psychology, 9: 43-56.

Kahneman, D. (1974). Reference points, anchors, norms, and mixed feelings. Special Issue: Decision processes in negotiation. Organizational Behavior \& Human Decision Processes, 51(2): 296-312.

Kahneman, D. (1982). Judgment and decision making: A personal view. Psychological Science, 2(3): 142-145. 